\title{
InGaAs/InP single-photon detector with low noise, low timing jitter and high count rate
}

\author{
Mirko Sanzaro, Niccolò Calandri, Alessandro Ruggeri, Carmelo Scarcella, Gianluca Boso, \\ Mauro Buttafava, Alberto Tosi* \\ Dipartimento di Elettronica, Informazione e Bioingegneria, Politecnico di Milano, \\ P.zza Leonardo da Vinci 32, I-20133 Milano, Italy
}

\begin{abstract}
We present a new InGaAs/InP Single-Photon Avalanche Diode (SPAD) with high detection efficiency and low noise, which has been employed in a sinusoidal-gated setup to achieve very low afterpulsing probability and high count rate.

The new InGaAs/InP SPAD has lower noise compared to previous generations thanks to the improvement of Zinc diffusion conditions and the optimization of the vertical structure. A detector with $25 \mu \mathrm{m}$ active-area diameter, operated in gated-mode with $\mathrm{ON}$ time of tens of nanoseconds, has a dark count rate of few kilo-counts per second at $225 \mathrm{~K}$ and $5 \mathrm{~V}$ of excess bias, $30 \%$ photon detection efficiency at $1550 \mathrm{~nm}$ and a timing jitter of less than $90 \mathrm{ps}$ (FWHM) at $7 \mathrm{~V}$ of excess bias.

In order to reduce significantly the afterpulsing probability, these detectors were operated with a sinusoidal gate at 1.3 GHz. The extremely short gate ON time (less than 200 ps) reduces the charge flowing through the junction, thus reducing the number of trapped carriers and, eventually, lowering the afterpulsing probability. The resulting detection system achieves a maximum count rate higher than $650 \mathrm{Mcount} / \mathrm{s}$ with an afterpulsing probability of about $1.5 \%$, a photon detection efficiency greater than $30 \%$ at $1550 \mathrm{~nm}$ and a temporal resolution of less than $90 \mathrm{ps}$ (FWHM).
\end{abstract}

Keywords: Single-photon avalanche diode (SPAD), APD, single photon, near-infrared, InGaAs/InP, gated mode, sinusoidal gating.

\section{INTRODUCTION}

A growing number of applications in the NIR (near-infrared) wavelength range from $1 \mu \mathrm{m}$ to $1.7 \mu \mathrm{m}$ need practical single-photon detectors that guarantee good performance.

In quantum cryptography, for example, fiber-based short-distance QKD systems need high-count-rate detectors to sustain high secret-bit rates. The ideal detector should also operate in free-running mode in order to avoid issues related to the synchronization between the transmitter and the receiver, which can lead to strong reduction of the system detection efficiency. Eye-safe time-of-flight laser ranging (LIDAR) and 3D imaging (Light Detection and Ranging) with gigahertz clock rates need high efficiency and high count rate detectors at $1550 \mathrm{~nm}$. Other applications include singletoxygen detection for dosimetry in PhotoDynamic Therapy (PDT) (where long time constants need to be reconstructed), time-resolved photoluminescence, optical time-domain reflectometry (OTDR), quantum experiments, integrated circuit characterization based on photon emission from hot-carriers in metal-oxide-semiconductor field-effect transistors.

An excellent choice for all these applications is the InGaAs/InP Single-Photon Avalanche Diode (SPAD), which requires moderate cooling and shows good features, but still needs some improvements to reduce noise. Devices with InGaAs absorption region and InP multiplication region proved to have the best performance, but must be generally used in gated-mode, with long hold-off period (up to few tens of microseconds). This technique is used to limit afterpulsing, which strongly depends on the avalanche charge.

Here we present a novel InGaAs/InP SPAD with low noise and good detection efficiency, which is operated with gigahertz sinusoidal gating in order to achieve high count rate and very low afterpulsing probability.

*alberto.tosi@polimi.it; phone+3902 2399.6174; fax +39022399.3699

Quantum Sensing and Nanophotonic Devices XII, edited by Manijeh Razeghi, Eric Tournié, Gail J. Brown, Proc. of SPIE Vol. 9370, 93701T · (C) 2015 SPIE · CCC code: 0277-786X/15/\$18 - doi: 10.1117/12.2079778 


\section{INGAAS/INP SPAD}

\subsection{Device structure and its improvements}

We designed, fabricated and characterized a planar InGaAs/InP SPAD based on the SAGCM (Separate Absorption Grading Charge and Multiplication) structure [1].

Compared to our previous runs, we improved the performance by tuning the $\mathrm{Zn}$ diffusion and its impact on the quality of the underlying layers. Zinc is diffused with dimethylzinc in a MOCVD (Metal-Organic Chemical Vapor Deposition) reactor and diffusion temperature, phosphine overpressure and dimethylzinc source flow have an impact on the concentration of mid-gap traps, introduced during epitaxial growth or successive processing, and associated with high dark current and noise [2].

The electric field in the multiplication layer was optimized for high PDE (Photon Detection Efficiency), low timing jitter and low noise. Indeed, high electric field gives high avalanche triggering probability and low timing jitter, but higher tunneling contribution to DCR [3]. Compared to our previous design, higher dose in the charge layer gives higher punchthrough voltage and lower electric field in the InGaAs layer.

\subsection{Experimental characterization}

For all the experimental characterization reported in this section, we operated the InGaAs/InP SPADs in standard square gated mode: the SPAD bias is $0.5 \mathrm{~V}$ below the breakdown voltage $\left(\mathrm{V}_{\mathrm{BD}} \sim 65 \mathrm{~V}\right)$ during the OFF time, while it is raised above the breakdown level (by an amount called excess bias, $\mathrm{V}_{\mathrm{EX}}$ ) during the $\mathrm{ON}$ time $\left(\mathrm{T}_{\mathrm{ON}}\right.$, lasting few tens of nanoseconds).

By enforcing a long hold-off time after each avalanche $\left(\mathrm{T}_{\mathrm{OFF}}>100 \mu \mathrm{s}\right)$, we measured the DCR of InGaAs/InP SPADs with different active area diameters, from $10 \mu \mathrm{m}$ to $100 \mu \mathrm{m}$. Figure 1 shows that DCR has a quadratic dependence with the active area diameter, while the measured dark current (not reported here) increases linearly with the diameter. This demonstrates that DCR is mainly due by carriers generated in the bulk, while peripheral leakage does not contribute to DCR, since such carriers do not succeed in triggering avalanche ignitions.

Figure 2 shows the dependence of DCR on temperature at six different excess bias voltages (from $2 \mathrm{~V}$ to $7 \mathrm{~V}$ ) of $25 \mu \mathrm{m}$ active area diameter SPAD: DCR is just few kcps at $225 \mathrm{~K}$. By decreasing temperature, the DCR decreases exponentially with a rate of about one decade every $25 \mathrm{~K}$ down to $225 \mathrm{~K}$. At lower temperatures, the slope is lower because of pile-up at hetero-interface of carriers thermally generated in the InGaAs layer during OFF time.

Figure 3 shows the afterpulsing probability measured by means of the double pulse method at $225 \mathrm{~K}$ with an excess bias of $5 \mathrm{~V}$. By fitting such decay, we extracted three time constants (about $9 \mu \mathrm{s}, 22 \mu \mathrm{s}$ and $322 \mu \mathrm{s}$ ), which roughly

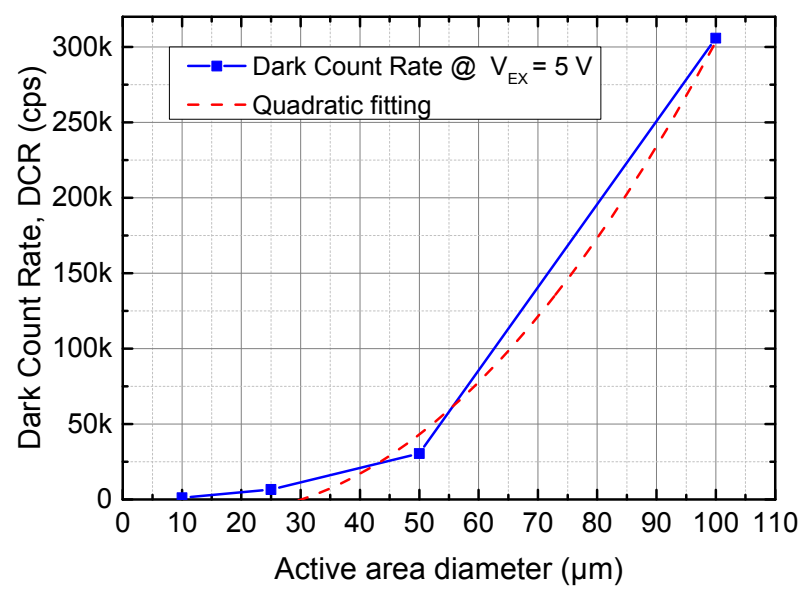

Figure 1: Primary dark count rate dependence on active area diameter at $225 \mathrm{~K}$ and with $\mathrm{V}_{\mathrm{EX}}=5 \mathrm{~V}$ (corresponding to $\mathrm{PDE}=28 \%$ at $1550 \mathrm{~nm}$ ). The DCR increases with the square of the active area diameter, whereas we measured that the dark current increases linearly with the active area diameter. 


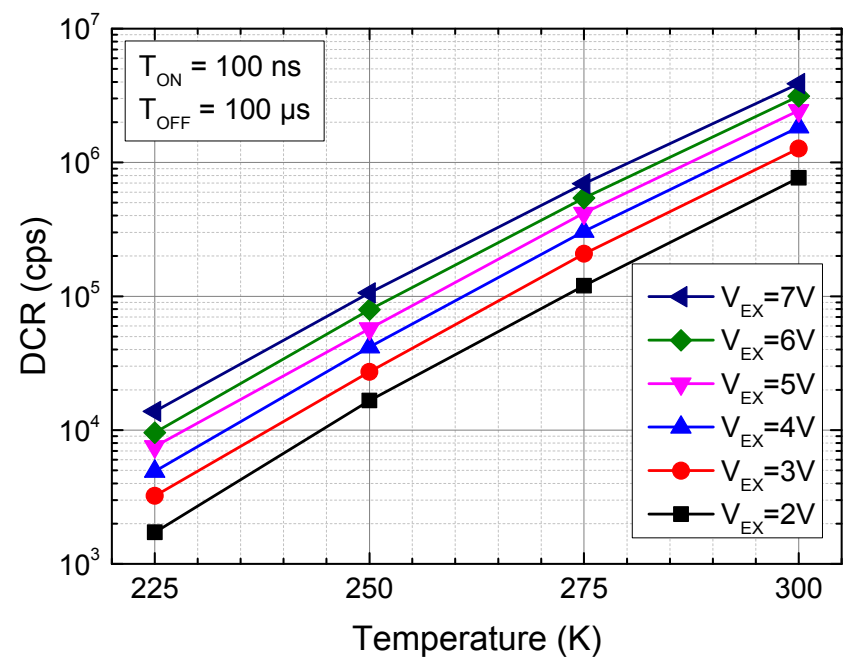

Figure 2: Primary DCR of the $25 \mu \mathrm{m}$ active area diameter InGaAs/InP SPAD as a function of temperature at six excess bias voltages from $2 \mathrm{~V}$ to $7 \mathrm{~V}$.

correspond to three deep levels families responsible for trapping of carriers during an avalanche. In comparison with the previous run [4] and other InGaAs/InP SPADs [5], afterpulsing is as good as state-of-the art detectors when operated in square gated mode, at this temperature and with $5 \mathrm{~V}$ excess bias voltage.

Figure 4 reports the relationship between DCR and PDE at different excess bias voltages. PDE from $20 \%$ to $30 \%$ can be achieved with DCR below $10 \mathrm{kcps}$, thus making these detectors one of the best InGaAs/InP SPADs ever reported in literature.

We measured the temporal response of the novel InGaAs/InP SPADs by illuminating it with a pulsed laser at $1550 \mathrm{~nm}$, whose pulse width is about 20 ps (FWHM), and acquiring the data with a standard Time-Correlated Single-Photon Counting (TCSPC) setup. Figure 5 shows the SPAD temporal response (after amplitude normalization) when operated with $\mathrm{V}_{\mathrm{EX}}=5 \mathrm{~V}$ : the FWHM is about 110 ps at $5 \mathrm{~V}$ excess bias, and it is even less than 90 ps at $7 \mathrm{~V}$ excess bias (not reported here). The temporal response is free from secondary peaks and bumps, with a main Gaussian distribution plus a fast exponentially decaying "tail" whose time constant is 66 ps at $\mathrm{V}_{\mathrm{EX}}=5 \mathrm{~V}$ and 58 ps at $\mathrm{V}_{\mathrm{EX}}=7 \mathrm{~V}$.

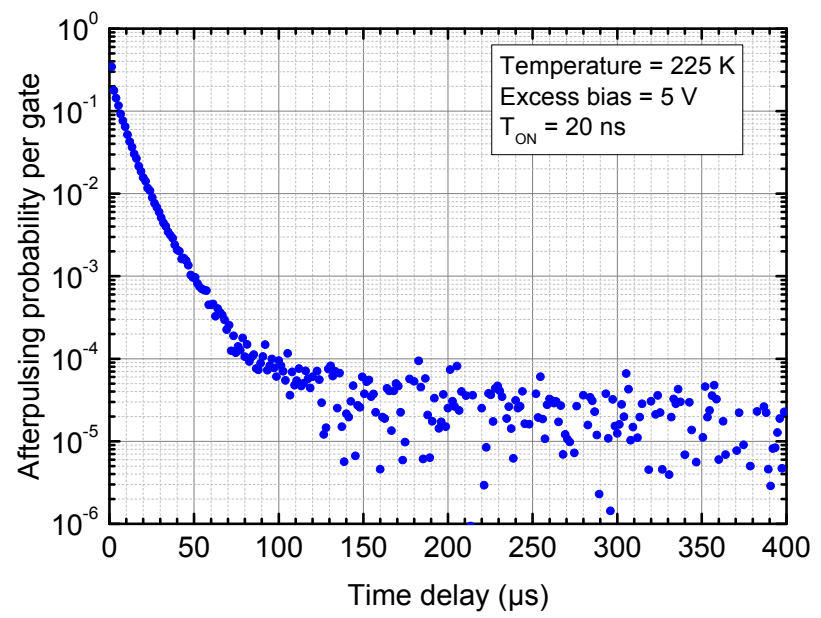

Figure 3: Afterpulsing probability per gate measured with the double pulse method $\left(\mathrm{T}_{\mathrm{ON}}=20 \mathrm{~ns}, \mathrm{~V}_{\mathrm{EX}}=5 \mathrm{~V}, \mathrm{~T}=225 \mathrm{~K}\right)$. 


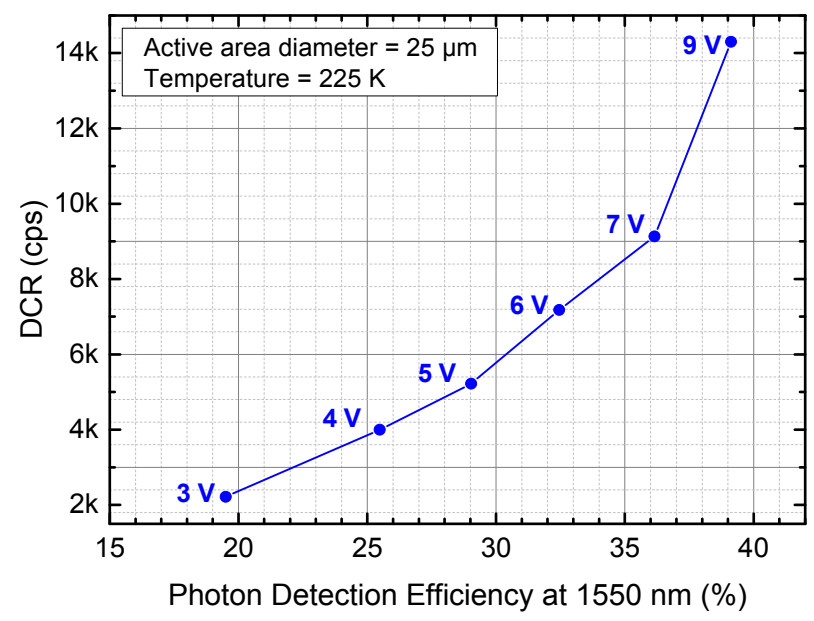

Figure 4: Primary Dark Count Rate at $225 \mathrm{~K}$ as a function of the Photon Detection Efficiency at $1550 \mathrm{~nm}$, with the corresponding excess bias voltages shown at each data point. Note that these data are taken from a SPAD different from the one of Figure 2.

\section{GIGAHERTZ SINUSOIDAL GATING}

\subsection{High-frequency balanced detector configuration}

InGaAs/InP SPADs can be operated with sub-nanosecond square-wave gate signals [6] or gigahertz sine-wave gating [7][8][9]. With these techniques such detectors can reach high count rates thanks to a strong reduction of the afterpulsing probability and hence of the required hold-off time. The SPAD is enabled by a sinusoidally-modulated bias voltage at a frequency up to few gigahertz. The very short ON times (less than $1 \mathrm{~ns}$ ) is exploited to lower the afterpulsing probability by reducing the avalanche charge. Typically, the gate signal has to be precisely synchronized with the arrival time of the photons, which arrive in bursts.

We recently demonstrated that if we unlock the detector gate from the trigger signal of the light source, photons from the optical waveform hit the detector in any possible bias state resulting in a flat response in time of the detector. After many periods, the SPAD samples uniformly the optical signal and the mean behavior is equivalent to the free-running mode,

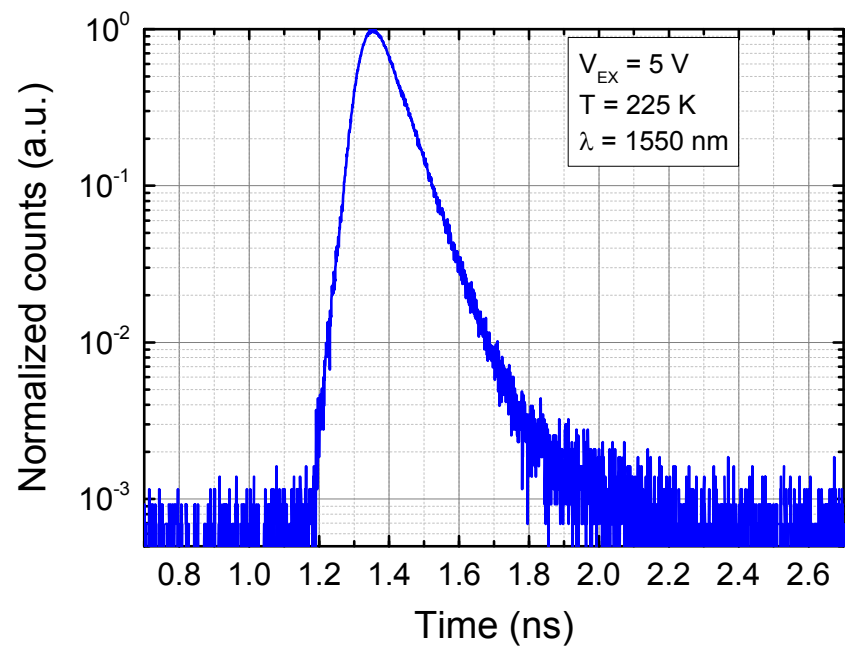

Figure 5: Temporal response to a $20 \mathrm{ps}$ pulsed laser at $1550 \mathrm{~nm}$ at $5 \mathrm{~V}$ excess bias voltage, when the InGaAs/InP SPAD is cooled at $225 \mathrm{~K}$. 


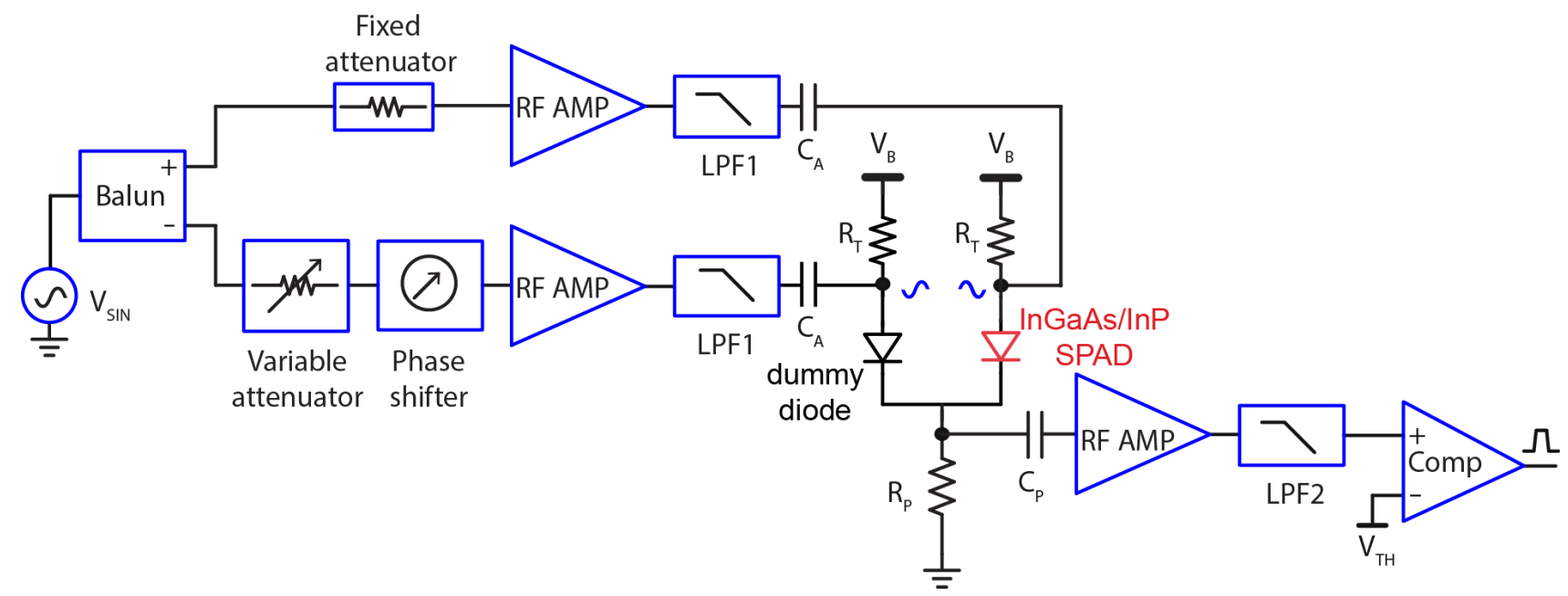

Figure 6 Simplified diagram of the experimental setup to cancel the capacitive coupling of the gate signal through the SPAD. RF AMP: wide-bandwidth amplifier. LPF1 = low-pass filter at $1.45 \mathrm{GHz}$. LPF2 = low-pass filter at $2.4 \mathrm{GHz}$. Comp $=$ wide-bandwidth comparator. $\mathrm{V}_{\mathrm{TH}}=$ threshold of the comparator.

where the detector is always ON except for a hold-off time [10]. This approach makes it possible to have a free-running equivalent InGaAs/InP detector with very low afterpulsing probability (thanks to the extremely short ON time, few hundreds of picoseconds).

Here we describe an improved version of such approach, where our InGaAs/InP SPAD is sinusoidal-gated at $\mathrm{f}_{\mathrm{GATE}}=$ $1.3 \mathrm{GHz}$. We exploited a SPAD-dummy balancing technique for cancelling the spurious capacitive coupling from the gate driver to the read-out circuit. Figure 6 shows the block diagram of our experimental setup. The SPAD is wirebonded to a passive-quenching front-end board and is cooled to $240 \mathrm{~K}$ with a four-stage thermo-electric cooler.

The input signal $\mathrm{V}_{\mathrm{SIN}}$ at $1.3 \mathrm{GHz}$ is split (balun) in two paths, phase shifted by $180^{\circ}$, and amplified by $33 \mathrm{~dB}$ (RF amplifiers). The two anti-phase signals are then fed to the SPAD and a dummy diode, both integrated in the same chip. The "dummy diode" mimics very well the parasitic capacitances and inductances of the SPAD, but no avalanche is triggered in it. The two diodes are both biased at the SPAD breakdown voltage (i.e. $\mathrm{V}_{\mathrm{B}} \sim 65 \mathrm{~V}$ ), the gate signals are applied at the anodes, while the avalanche signal is picked-up from the common cathode node. The SPAD is periodically biased above its breakdown voltage by the gate signal. With this structure, the SPAD gate feed-through is balanced with its own anti-phase copy propagating through the dummy diode.

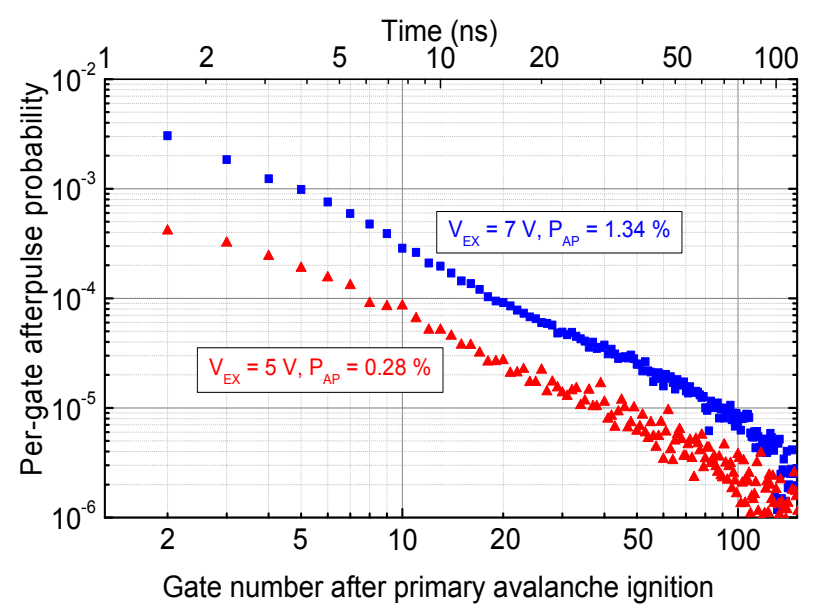

Figure 7 Per-gate afterpulsing probability as a function of number of gates after primary avalanche ignition. The total afterpulsing probability $\left(\mathrm{P}_{\mathrm{AP}}\right)$ is calculated integrating the per-gate afterpulsing probability. 


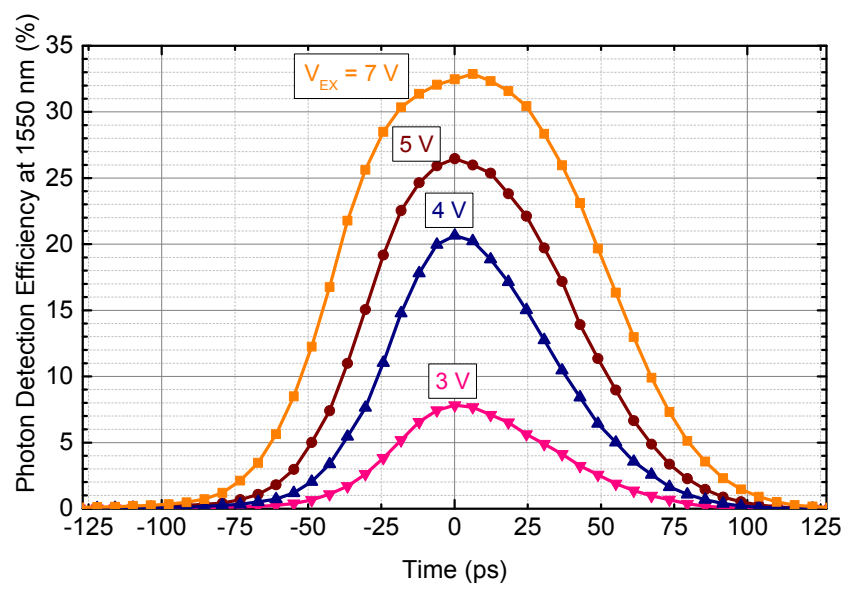

Figure 8 Photon detection efficiency at $\lambda=1550 \mathrm{~nm}$ within the gate at four different excess bias voltages.

An RF amplifier is AC coupled to the readout node and amplifies the avalanche signal by $20 \mathrm{~dB}$, and then a fast comparator extracts a digital pulse. A monostable is included in the comparator to provide a fixed-duration output pulse and hence to implement a count-off time after each avalanche.

The symmetry of the SPAD-“dummy diode" approach suppresses the gate feed-through spurious signals, but component tolerances may introduce some residual mismatches, which can be further reduced by fine-tuning the amplitude and phase of the gate signal with the voltage-controlled variable attenuator and variable phase shifter.

\subsection{Experimental results}

The DCR is not affected by the gating approach and it depends on the quality of the SPAD itself (see Figure 2 and previous sections). This is guaranteed also by the low threshold of the read-out comparator that detects even small avalanches triggered when the excess bias is low.

The afterpulsing probability has been characterized with the Time-Correlated Carrier Counting (TCCC) technique. Figure 7 shows the per-gate afterpulsing probability at two excess-bias voltages. Afterpulses have been recorded starting from the second gate after the avalanche, since the first one is masked by the count-off time given by the monostable of the fast comparator. A total afterpulsing probability is calculated by integrating the curves and results $1.34 \%$ at $\mathrm{V}_{\mathrm{EX}}=$ $7 \mathrm{~V}$, while it is even below $0.3 \%$ at $\mathrm{V}_{\mathrm{EX}}=5 \mathrm{~V}$.

We measured the system Photon Detection Efficiency (PDE) at $\lambda=1550 \mathrm{~nm}$ within the gate by acquiring the arrival times of the triggered avalanche pulses with a Time-Correlated Single-Photon Counting (TCSPC) board. Figure 8 shows

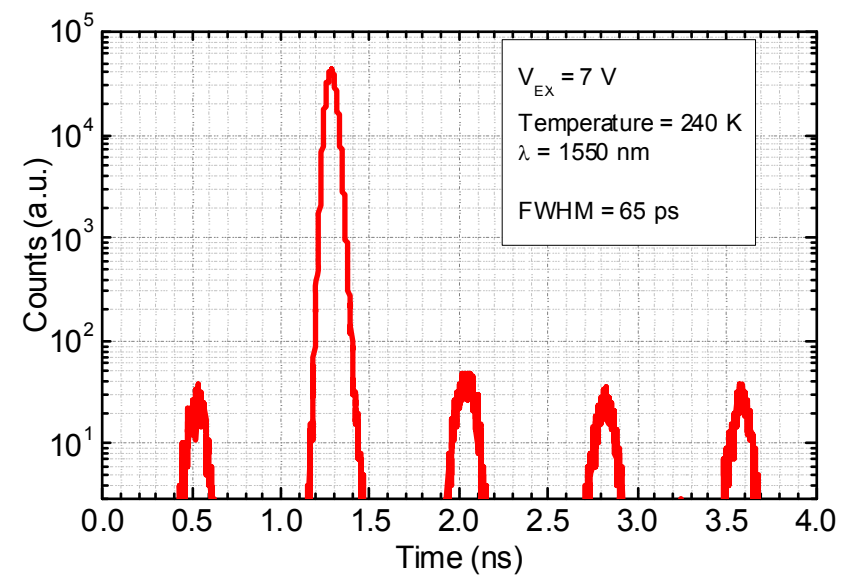

Figure 9 Temporal response of the InGaAs/InP SPAD when sinusoidally gated at $1.3 \mathrm{GHz}$, with the laser source synchronous with the gate waveform. 
the time distribution of the triggered avalanches within the gate, with a maximum PDE at the center of the gate of more than $30 \%$ at $\mathrm{V}_{\mathrm{EX}}=7 \mathrm{~V}$. The FWHM of the distribution is about $100 \mathrm{ps}$.

We measured the response of the sinusoidally-gated system with the $1550 \mathrm{~nm}$ laser pulses synchronized with the $1.3 \mathrm{GHz}$ gate frequency (see Figure 9) and with the laser pulses unlocked from the gate waveform. For both measurements, the InGaAs/InP SPAD is operated with $\mathrm{V}_{\mathrm{EX}}=7 \mathrm{~V}$. The timing jitter is very low, being just $65 \mathrm{ps}$ (FWHM) with synchronous laser pulses, and increases to about $90 \mathrm{ps}$ with asynchronous laser pulses. The small peaks in Figure 9 are due to dark counts and background photons triggering avalanches in all the gates.

Finally, we characterized the linearity of the response to a light stimulus by increasing the illuminating photon flux until saturation is reached. With just $1.5 \mathrm{~ns}$ of count-off time, only a single gate after each avalanche detection is skipped and it results in a maximum count rate of about $650 \mathrm{Mcps}$.

\section{CONCLUSIONS}

We presented a novel InGaAs/InP Single-Photon Avalanche Diode (SPAD) with high detection efficiency and low noise, which has been sinusoidal-gated at $1.3 \mathrm{GHz}$ to achieve very low afterpulsing probability and high count rate.

A $25 \mu \mathrm{m}$ active area diameter InGaAs/InP SPAD has a dark count rate of few kilo-counts per second at $225 \mathrm{~K}$ and $5 \mathrm{~V}$ of excess bias, $30 \%$ photon detection efficiency at $1550 \mathrm{~nm}$ and a timing jitter of less than $90 \mathrm{ps}$ (FWHM) at $7 \mathrm{~V}$ of excess bias. When operated with a sinusoidal gate at $1.3 \mathrm{GHz}$, the short gate $\mathrm{ON}$ time reduces the afterpulsing probability. The resulting system proved to reach a maximum count rate of about $650 \mathrm{Mcps}$ with an afterpulsing probability of about $1.5 \%$, a photon detection efficiency greater than $30 \%$ at $1550 \mathrm{~nm}$ and a temporal resolution of less than $90 \mathrm{ps}$ (FWHM).

\section{ACKNOWLEDGEMENTS}

Authors wish to thank Franco Zappa and Fabio Acerbi for their useful discussions.

\section{REFERENCES}

[1] Tosi, A., Calandri, N., Sanzaro, M., Acerbi, F., "Low-Noise, Low-Jitter, High Detection Efficiency InGaAs/InP Single-Photon Avalanche Diode," IEEE J. Sel. Top. Quantum Electron. 20(6), 1-6 (2014).

[2] Sussmann, R. S., Ash, R. M., Murphy, A., Monham, K. L., "Dark current processes in InP/GaInAs heterostructure APDs," Phys. B+C 129(1), 473-477 (1985).

[3] Acerbi, F., Anti, M., Tosi, A., Zappa, F., "Design Criteria for InGaAs/InP Single-Photon Avalanche Diode," IEEE Photonics J. 5(2), 6800209-6800209 (2013).

[4] Tosi, A., Acerbi, F., Anti, M., Zappa, F., "InGaAs/InP Single-Photon Avalanche Diode With Reduced Afterpulsing and Sharp Timing Response With 30 ps Tail," IEEE J. Quantum Electron. 48(9), 1227-1232 (2012).

[5] Itzler, M. A., Jiang, X., Entwistle, M., lomkowski, K., Tosi, A., Acerbi, F., Zappa, F., Cova, S., "Advances in InGaAsP-based avalanche diode single photon detectors," Journal of Modern Optics, 58(3-4), 174-200 (2011).

[6] Restelli, A., Bienfang, J. C., Migdall, A. L., "Time-domain measurements of afterpulsing in InGaAs/InP SPAD gated with sub-nanosecond pulses," Journal of Modern Optics, 59(17), 1465-1471 (2012).

[7] Namekata, N., Adachi, S., Inoue, S., "Ultra-Low-Noise Sinusoidally Gated Avalanche Photodiode for High-Speed Single-Photon Detection at Telecommunication Wavelengths," IEEE Photonics Technology Letters, 22(8), 529531 (2010).

[8] Patel, K. A., Dynes, J. F., Sharpe, A. W., Yuan, Z. L., Penty, R. V., Shields, A. J., "Gigacount/second photon detection with InGaAs avalanche photodiodes," Electronics Letters, 48(2), 111 (2012).

[9] Zhang, J., Eraerds, P., Walenta, N., Barreiro, C., Thew, R., Zbinden, H., "2.23 GHz gating InGaAs/InP singlephoton avalanche diode for quantum key distribution," in Proc. SPIE 7681, Advanced Photon Counting Techniques IV, 7681, 76810Z-76810Z-8 (2010).

[10] Tosi, A., Scarcella, C., Boso, G., Acerbi, F., "Gate-Free InGaAs/InP Single-Photon Detector Working at Up to 100 Mcount/s,” IEEE Photonics J. 5(4), 6801308-6801308 (2013). 